\title{
Tail Estimates for One-Dimensional Random Walk in Random Environment
}

\author{
Amir Dembo $^{1 . *}$, Yuval Peres ${ }^{2, * *}$, Ofer Zeitouni ${ }^{3, * * *}$ \\ ${ }^{1}$ Department of Mathematics and Department of Statistics, Stanford University, Stanford, CA 94305 \\ and Department of Electrical Engineering, Technion, Israel Institute of Technology, Haifa 32000, \\ ISRAEL. \\ ${ }^{2}$ Department of Statistics, University of California, Berkeley, California 94720 and Institute of \\ Mathematics, Hebrew University, Jerusalem. \\ E-mail: peres@math.huji.ac.il \\ ${ }^{3}$ Department of Electrical Engineering, Technion, Israel Institute of Technology, Haifa 32000, \\ ISRAEL.
}

Received: 15 November 1995/Accepted: 21 April 1996

\begin{abstract}
Suppose that the integers are assigned i.i.d. random variables $\left\{\omega_{x}\right\}$ (taking values in the unit interval), which serve as an environment. This environment defines a random walk $\left\{X_{k}\right\}$ (called a RWRE) which, when at $x$, moves one step to the right with probability $\omega_{x}$, and one step to the left with probability $1-\omega_{x}$. Solomon (1975) determined the almost-sure asymptotic speed (= rate of escape) of a RWRE. For certain environment distributions where the drifts $2 \omega_{x}-1$ can take both positive and negative values, we show that the chance of the RWRE deviating below this speed has a polynomial rate of decay, and determine the exponent in this power law; for environments which allow only positive and zero drifts, we show that these large-deviation probabilities decay like $\exp \left(-C n^{1 / 3}\right)$. This differs sharply from the rates derived by Greven and den-Hollander (1994) for large deviation probabilities conditioned on the environment. As a by product we also provide precise tail and moment estimates for the total population size in a Branching Process with Random Environment.
\end{abstract}

\section{Introduction}

In this paper we consider the large deviations of the position of a nearest-neighbor random walk on $\mathbb{Z}$ with site-dependent transition probabilities.

Let $\omega=\left(\omega_{x}\right)_{x \in \mathbb{Z}}$ be an i.i.d. collection of $(0,1)$-valued random variables, with marginal distribution $\alpha$ such that supp $\alpha \subset(0,1)$. For every fixed $\omega$, let $X=\left(X_{n}\right)_{n} \geqq 0$

\footnotetext{
* Partially supported by NSF DMS-9209712 and DMS-9403553 grants, by a US-ISRAEL BSF grant and by the S. and N. Grand research fund.

** Research partially supported by NSF grant \# DMS-9404391 and a Junior Faculty Fellowship from the Regents of the University of California.

*** Partially supported by NSF grant \# DMS-9302709, by a US-Israel BSF grant and by the fund for promotion of research at the Technion.
} 
be the Markov chain on $\mathbb{Z}$ starting at $X_{0}=0$ (unless explicitly stated otherwise), and with transition probabilities

$$
\mathbf{P}_{\omega}\left(X_{n+1}=y \mid X_{n}=x\right)=\left\{\begin{array}{cl}
\omega_{x} & \text { if } y=x+1 \\
1-\omega_{x} & \text { if } y=x-1 \\
0 & \text { otherwise }
\end{array}\right.
$$

The symbol $\mathbf{P}_{\omega}$ denotes the measure on path space given by the environment $\omega$. The process $(X, \omega)$ is an example of a random walk in random environment (RWRE), and $X$ has law $\mathbf{P}=\int \alpha^{\mathbb{Z}}(d \omega) \mathbf{P}_{\omega}$.

This model exhibits a number of phenomena not shared by the classical random walk.

Abbreviate $\rho=\rho(x, \omega)=\left(1-\omega_{x}\right) / \omega_{x}$ and $\langle f\rangle=\int f(\omega) \alpha^{\mathbb{Z}}(d \omega)$ for any function $f$ of the environment. It was established by Solomon [11] that $X$ is $\omega$-a.s. transient iff $\langle\log \rho\rangle \neq 0$. In the transient case $\lim _{n \rightarrow \infty} X_{n}=+\infty \mathbf{P}$-a.s. if $\langle\log \rho\rangle<0$ (and $\lim _{n \rightarrow \infty} X_{n}=-\infty$ P-a.s. if $\langle\log \rho\rangle>0$ ). With $v_{\alpha}=\lim _{n \rightarrow \infty} n^{-1} X_{n}$ denoting the P-a.s. speed of the RWRE, there are two speed regimes, namely,

(i) $v_{\alpha}=(1-\langle\rho\rangle) /(1+\langle\rho\rangle)$ when $\langle\rho\rangle<1$ and $v_{\alpha}=\left(\left\langle\rho^{-1}\right\rangle-1\right) /\left(\left\langle\rho^{-1}\right\rangle+1\right)$ when $\left\langle\rho^{-1}\right\rangle<1$. (See Remark 2.1 for a transparent derivation of this result.)

(ii) $v_{\alpha}=0$ when $\langle\rho\rangle^{-1} \leqq 1 \leqq\left\langle\rho^{-1}\right\rangle$.

This law of large numbers is supplemented in [8] by central limit type theorems. For instance, in regime (i) the classical central limit theorem holds if $\left\langle\rho^{2}\right\rangle<1$. In regime (ii), on the other hand, if $\langle\log \rho\rangle<0<\log \langle\rho\rangle$ then $n^{-s} X_{n}$ converges in law with $s \in(0,1)$ the unique solution of $\left\langle\rho^{s}\right\rangle=1$.

In the recurrent case the motion is extremely slow. Sinai [10] proved that in this case $(\log n)^{-2} X_{n}$ converges in law, and Kesten [7] identified explicitly the limiting law.

The large deviations of $n^{-1} X_{n}$ in the quenched setting, namely, conditional on the environment, are derived in [5]. Specifically, the limit $I(v)$ of $-n^{-1} \log \mathbf{P}_{\omega}\left(X_{n}=\right.$ $\lfloor v n\rfloor)$ is characterized as the solution to a variational problem involving specific relative entropy with respect to a certain stationary Markov process.

In this paper, we study the large deviations of $n^{-1} X_{n}$ in the annealed setting, namely averaging over environments $\omega$ according to the measure $\alpha^{\mathbb{Z}}$. We shall assume hereafter that $\langle\rho\rangle<1$, hence the RWRE is transient and of strictly positive speed $v_{\alpha}$ (the case $\left\langle\rho^{-1}\right\rangle<1$ follows by reflection).

How can the walk deviate significantly from its almost-sure limiting speed $v_{\alpha}$ ? For ordinary $\mathrm{RW}$, this is exponentially unlikely and given that such a deviation has occurred, it is most likely to arise from movement at an approximately constant different speed. For RWRE there are other possibilities-large deviations can arise from relatively short, atypical segments in the environment.

The next two theorems are our main results, characterizing the subexponential slow-down probabilities $\mathbf{P}\left(n^{-1} X_{n} \in G\right)$ in the mixed-drift cases for any open $G \subset\left(0, v_{\alpha}\right)$ which is separated from $v_{\alpha}$. A polynomial rate of decay is obtained when a negative local drift is possible, whereas for environments which allow only positive and zero drifts, the large-deviation slow-down probabilities decay like $\exp \left(-C n^{1 / 3}\right)$.

Let $\rho_{\max }$ denote the supremum of $\rho$ over the closed support of $\alpha$, and let $\rho_{\min }$ denote the corresponding minimum. 
Theorem 1.1 (Positive and negative drifts). Suppose that $\langle\rho\rangle\left\langle 1\right.$ and $\infty>\rho_{\max }>1$. Then, there exists a unique $s>1$ satisfying $\left\langle\rho^{s}\right\rangle=1$ such that for any open $G \subset$ $\left(0, v_{\alpha}\right)$ which is separated from $v_{\alpha}$,

$$
\lim _{n \rightarrow \infty} \log \mathbf{P}\left(n^{-1} X_{n} \in G\right) / \log n=1-s .
$$

The condition $\rho_{\max }<\infty$ can be relaxed (see Remark 5 in Sect. 6).

Theorem 1.2 (Positive and zero drifts). Suppose that $\langle\rho\rangle<1$, but $\rho_{\max }=1$ and $\alpha(\{1 / 2\})>0$. Then, for any open $G \subset\left(0, v_{\alpha}\right)$ which is separated from $v_{\alpha}$,

$$
-\infty<\liminf _{n \rightarrow \infty} n^{-1 / 3} \log \mathbf{P}\left(n^{-1} X_{n} \in G\right) \leqq \limsup _{n \rightarrow \infty} n^{-1 / 3} \log \mathbf{P}\left(n^{-1} X_{n} \in G\right)<0 .
$$

The next proposition complements Theorems 1.1 and 1.2 , by showing that the large-deviation probabilities outside $\left(0, v_{\alpha}\right)$ always decay exponentially. If all drifts in the environment are in the same direction and bounded away from 0 , then any deviation from the limiting speed $v_{\alpha}$ is exponentially unlikely.

Proposition 1.3. For $\langle\rho\rangle<1$ and any closed set $F$ which is disjoint from $\left[0, v_{\alpha}\right]$,

$$
\limsup _{n \rightarrow \infty} n^{-1} \log \mathbf{P}\left(n^{-1} X_{n} \in F\right)<0 \text {. }
$$

If also $\rho_{\max }<1$ then (2) holds for any closed $F$ that does not contain $v_{\alpha}$.

The above results cover in particular all cases in which the RWRE has a positive speed and the marginal environment distribution $\alpha$ is finitely supported. (Other cases are described in the last section.)

Sections 2, 4 and 5 contain the proofs of Theorems 1.1, 1.2 and Proposition 1.3 respectively, while Sect. 6 discusses some variants and unsolved problems. We note that the upper bounds in Theorems 1.1 and 1.2 are harder to prove than the lower bounds. Indeed the lower bound in Theorem 1.2 is straightforward; establishing that it is sharp was the initial impetus for our work. The key to our upper bounds is Lemma 2.4. As explained in Sect. 3, this lemma applies in a wider context, supplying also precise tail and moment estimates on the total population size of a Branching Process in Random Environment (called BPRE).

\section{Positive and Negative Drifts: Proof of Theorem 1.1}

Throughout this section, the hypotheses of Theorem 1.1 are in force. Since $\rho_{\max }>1$, the convex, continuous map $\lambda \mapsto\left\langle\rho^{\lambda}\right\rangle$ from $\mathbb{R}_{+}$to $\mathbb{R}_{+}$satisfies $\lim _{\lambda \rightarrow \infty}\left\langle\rho^{\lambda}\right\rangle=\infty$. Moreover, $\langle\rho\rangle<1$, yielding both the existence and uniqueness of $s>1$ such that $\left\langle\rho^{s}\right\rangle=1$. We fix this value of $s$ for the rest of the section.

Let $\bar{X}_{n}$ denote the Markov chain, initialized at zero, with the same $\omega$-dependent transition kernel as in (1) but now with the value of $\omega_{0}$ set to be $\omega_{0}=1$. Let

$$
\bar{\tau}_{k}=\inf \left\{n: \bar{X}_{n}=k\right\}
$$

and

$$
R_{k}(m)=k^{-1} \sum_{i=m+1}^{m+k} \log \rho(i), \quad \text { with } R_{k}=R_{k}(0) .
$$

The following simple tail estimate for $\bar{\tau}_{k}$ is used in the proof of the lower bound on $\mathbf{P}\left(n^{-1} X_{n} \in G\right)$. 
Lemma 2.1. For all $n$ and $k$,

$$
\mathbf{P}_{\omega}\left(\bar{\tau}_{k} \geqq n\right) \geqq\left(1-e^{-(k-1) R_{k-1}}\right)^{n} .
$$

Proof. Let $\sigma_{k}=\inf \left\{n \geqq 1: \bar{X}_{n}=k\right.$ or $\left.\bar{X}_{n}=0\right\}$. By Chung [2, pp. 65-71], we have

$$
\mathbf{P}_{\omega}\left(\bar{X}_{\sigma_{k}}=0\right)=1-\left(\sum_{i=0}^{k-1} e^{i R_{i}}\right)^{-1} \geqq\left(1-e^{-(k-1) R_{k-1}}\right),
$$

and the lower bound follows since $\mathbf{P}_{\omega}\left(\bar{\tau}_{k} \geqq n\right) \geqq \mathbf{P}_{\omega}\left(\bar{X}_{\sigma_{k}}=0\right)^{n}$.

For $y \in \mathbb{Z}$, let $\tau_{y}=\min \left\{n: X_{n}=y\right\}$. The next lemma provides an exponential tail estimate on $L_{y}=\max \left\{y-X_{n}: n \geqq \tau_{y}\right\}$, the longest excursion of the RWRE path to the left of $y$. This estimate is crucial to the proof of both the upper and the lower bounds on $\mathbf{P}\left(n^{-1} X_{n} \in G\right)$.

Lemma 2.2. For every $y \in \mathbb{Z}$ and any $k \geqq 1$,

$$
\mathbf{P}\left(L_{y} \geqq k\right) \leqq \frac{\langle\rho\rangle^{k}}{1-\langle\rho\rangle} .
$$

Proof. Note that for each $k \geqq 1$,

$$
\Theta_{y, k} \triangleq \mathbf{P}_{\omega}\left(L_{y} \geqq k\right)=\mathbf{P}_{\omega}\left(X_{n}=y-k \text { for some } n>0 \mid X_{0}=y\right)
$$

is a stationary process (when $\omega$ has the distribution $\alpha^{\mathbb{Z}}$ ). By, e.g., [2, pp. 65-71],

$$
\Theta_{0, k}=\frac{\sum_{i=0}^{\infty} \prod_{x=-(k-1)}^{i} \rho(x)}{1+\sum_{i=-(k-1)}^{\infty} \prod_{x=-(k-1)}^{i} \rho(x)} \leqq \sum_{i=0}^{\infty} \prod_{x=-(k-1)}^{i} \rho(x)=Z .
$$

The lemma follows by observing that $\mathbf{E}(Z)=\sum_{i=0}^{\infty}\langle\rho\rangle^{i+k}$ while $\mathbf{P}\left(L_{y} \geqq k\right)=$ $\mathbf{E}\left(\Theta_{y, k}\right)$.

Since $G \subset\left(0, v_{\alpha}\right)$ is open and separated from $v_{\alpha}$, it suffices to establish the lower bound for $G=(v-2 \eta, v)$ as in the next lemma.

Lemma 2.3. Let $0<2 \eta<v<v_{\alpha}$. Then

$$
\liminf _{n \rightarrow \infty} \log \mathbf{P}\left(n^{-1} X_{n} \in(v-2 \eta, v)\right) / \log n \geqq 1-s .
$$

Proof. Fix $v$ and $\eta$ as in the statement of the lemma. Observe that the event $n^{-1} X_{n} \in(v-2 \eta, v)$ contains the event

$$
\left\{\frac{(v-2 \eta) n}{v_{\alpha}}<\tau_{(v-\eta) n}<n \text {, the excursion distance } L_{(v-\eta) n}<\eta n \text {, and } \tau_{v n}>n\right\} \text {, }
$$

namely, that the RWRE hits $(v-\eta) n$ at about the expected time, from which point its longest excursion to the left is less than $\eta n$, but the RWRE does not arrive at position $v n$ by time $n$. Recall that $\mathbf{P}\left(\tau_{(v-\eta) n} \in\left((v-2 \eta) n / v_{\alpha}, n\right)\right) \rightarrow 1$ as $n \rightarrow \infty$ 
(by Solomon [11, Theorem 1.16]), and set $\xi=1-(v-2 \eta) / v_{\alpha}>0$. Since $\tau_{(v-\eta) n}$ is independent of $\left\{\omega_{x}: x \geqq(v-\eta) n\right\}$, it follows by stationarity that

$$
\mathbf{P}\left(\tau_{v n}>n \mid \tau_{(v-\eta) n} \in\left((v-2 \eta) n / v_{\alpha}, n\right)\right) \geqq \mathbf{P}\left(\bar{\tau}_{\eta n}>\xi n\right) .
$$

Hence, by the exponential bound on $\mathbf{P}\left(L_{(v-\eta) n} \geqq \eta n\right)$ (see Lemma 2.2), we establish (6) as soon as we show that

$$
\liminf _{n \rightarrow \infty} \log \mathbf{P}\left(\bar{\tau}_{\eta n}>\xi n\right) / \log n \geqq 1-s .
$$

To this end, let $y_{\delta}=\left\langle\rho^{s-\delta} \log \rho\right\rangle /\left\langle\rho^{s-\delta}\right\rangle$ for $\delta \geqq 0$, and note that $y_{0}>0>\langle\log \rho\rangle$ by convexity of $x \mapsto x \log x$. Hence, for every $\delta>0$ small enough $y_{\delta}$ is finite and positive.

Applying Cramér's theorem to the i.i.d. real-valued random variables $\{\log \rho(x)\}_{x \in \mathbb{Z}}$ gives

$$
\liminf _{k \rightarrow \infty} \frac{1}{k} \log \mathbf{P}\left(R_{k-1} \geqq y_{\delta}\right)=-y_{\delta} s_{\delta},
$$

where $s_{\delta}=s-\delta-\left(y_{\delta}\right)^{-1} \log \left\langle\rho^{s-\delta}\right\rangle$ (see, e.g., [4, Corollary 2.2.19 and Lemma 2.2.5]). In other words,

$$
\mathbf{P}\left(R_{k-1} \geqq y_{\delta}\right) \geqq e^{-\left(s_{\delta} y_{\delta}+o(1)\right) k} \quad \text { as } k \rightarrow \infty
$$

Choose

$$
k=k(n)=1+\frac{\log n}{y_{\delta}},
$$

so that $e^{-\left(s_{\delta} y_{\delta}+o(1)\right) k}=n^{-s_{\delta}+o(1)}$ as $n$ and $k$ tend to $\infty$, and consider the event

$$
\mathscr{A}_{n}=\left\{\omega: \max _{m=0,1, \ldots,[\eta n / k]-1} R_{k-1}(m k, \omega) \geqq y_{\delta}\right\} \text {. }
$$

Since $\left\{R_{k-1}(m k)\right\}_{m \geqq 0}$ are i.i.d. variables, we obtain from (8) that

$$
\liminf _{n \rightarrow \infty} \frac{1}{\log n} \log \mathbf{P}\left(\mathscr{A}_{n}\right) \geqq \liminf _{n \rightarrow \infty} \frac{1}{\log n} \log \left(\frac{\eta n}{k(n)} n^{-s_{\delta}}\right)=1-s_{\delta} .
$$

Decomposing the event $\mathscr{A}_{n}$ according to $m^{*}=\min \left\{m \geqq 0: R_{k-1}(m k) \geqq y\right\}$ and ignoring the time which the chain $X_{n}$ spends outside $\left[m^{*} k, m^{*} k+k\right)$, we get by stationarity that

$$
\mathbf{P}\left(\bar{\tau}_{\eta n}>\xi n \mid \mathscr{A}_{n}\right) \geqq \inf _{\omega: R_{k-1}(\omega) \geqq y_{\delta}} \mathbf{P}_{\omega}\left(\bar{\tau}_{k}>\xi n\right) .
$$

By Lemma 2.1,

$$
\inf _{\omega: R_{k-1}(\omega) \geqq y_{\delta}} \mathbf{P}_{\omega}\left(\bar{\tau}_{k}>\xi n\right) \geqq \inf _{z \geqq y_{\delta}}\left(1-e^{-(k-1) z}\right)^{(\xi n+1)} \geqq\left(1-n^{-1}\right)^{(\xi n+1)} .
$$

Combining (9) and (10) and taking $\delta \downarrow 0$ (for which $s_{\delta} \rightarrow s$ ), we establish (7), thus completing the proof of the lemma. 
The upper bound on $\mathbf{P}\left(n^{-1} X_{n} \in G\right)$ hinges upon moment estimates on the hitting times $\tau_{k}$. To derive these observe that $\tau_{k}=\sum_{i=1}^{k}\left(\tau_{i}-\tau_{i-1}\right)$ (with $\tau_{0}=0$ ), is the sum of the identically distributed, (dependent) random variables $\left(\tau_{i}-\tau_{i-1}\right)$, the law of each of which is identical to the law of $\tau_{1}$. Let $C_{\gamma}=\mathbf{E}\left(\tau_{1}^{\gamma}\right)$ and note that by Minkowski's inequality for all $k \geqq 1$,

$$
\mathbf{E}\left(\tau_{k}^{\gamma}\right) \leqq C_{\gamma} k^{\gamma}
$$

Our goal is thus to prove that $C_{\gamma}<\infty$ for all $\gamma<s$.

To this end, let $W_{0}=1$ and for negative integers $x$, let $W_{x}$ be the cardinality of the finite set $\mathscr{S}_{x}=\left\{n \in\left\{1, \ldots, \tau_{1}\right\}: X_{n-1}=x, X_{n}=x+1\right\}$. Then, $\tau_{1}=2 Z-1$ for the integer valued

$$
Z=\sum_{x=-\infty}^{0} W_{x}
$$

With $n_{0}=0$ and $\mathscr{S}_{x}=\left\{n_{1}<n_{2}<\cdots<n_{W_{x}}\right\}$ we have the representation

$$
W_{x-1}=\sum_{i=1}^{W_{x}} N_{x}^{(i)}
$$

where $N_{x}^{(i)}$ is the number of excursions to the left of $x$ (each starting and ending at $x$ ), during the time interval $\left\{n_{i-1}, \ldots, n_{i}\right\}$. The key observation is that the random variables $N_{x}^{(i)}$, for $x=0,-1, \ldots$ and $i=1,2, \ldots$ are independent under the measure $\mathbf{P}_{\omega}$ with

$$
\mathbf{P}_{\omega}\left(N_{x}^{(i)}=m\right)=\left(1-\omega_{x}\right)^{m} \omega_{x} \quad \forall m \in \mathbb{Z}_{+} .
$$

Denote $N_{x}=N_{x}^{(1)}$. In particular, $\mathbf{E}_{\omega}\left(N_{x}\right)=\rho(x, \omega)$ so that for every $\gamma \geqq 0$

$$
\mathbf{E}\left[\left(\mathbf{E}_{\omega} N_{x}\right)^{\gamma}\right]=\left\langle\rho^{\gamma}\right\rangle .
$$

Remark 2.1. The representation (12) yields the following transparent derivation of Solomon's formula for the limiting speed $v_{\alpha}$. Since $\left\{N_{x}^{(i)}\right\}_{i}$ are non-negative, identically distributed, and independent of $W_{x}$, we deduce from (13) that $\mathbf{E}\left(W_{x-1}\right)=$ $\mathbf{E}\left(W_{x}\right) \mathbf{E}\left(N_{x}\right)$. Since $W_{0}=1$ and $\mathbf{E}\left(N_{x}\right)=\langle\rho\rangle<1$, we conclude by monotone convergence that

$$
\mathbf{E}(Z)=\sum_{x=-\infty}^{0} \mathbf{E}\left(W_{x}\right)=\sum_{x=-\infty}^{0}\langle\rho\rangle^{-x}=(1-\langle\rho\rangle)^{-1}<\infty .
$$

The speed $v_{\alpha}$ is now computed from (15), since $v_{\alpha}=1 / \mathbf{E}\left(\tau_{1}\right)=1 /(2 \mathbf{E}(Z)-1)$ (cf. [11, Theorems 1.15, 1.16]).

We deduce next that $C_{\gamma}$ of (11) is finite for all $\gamma<s$.

Lemma 2.4. $\mathbf{E}\left(Z^{\gamma}\right)$ is finite for every $\gamma<s$.

Proof. Without loss of generality, we consider hereafter $\gamma \geqq 1$. Fix $\gamma \in[1, s), \varepsilon>0$ and a non-positive integer $x$. Let $c_{\gamma, \varepsilon}=\left(1-(1+\varepsilon)^{-1 / \gamma}\right)^{-\gamma}$. Since $z^{\gamma} \leqq(1+\varepsilon) y^{\gamma}+$ 
$c_{\gamma, \varepsilon}|z-y|^{\gamma}$ for every $z, y \geqq 0$, it follows from (13) that

$$
\mathbf{E}\left\{W_{x-1}^{\gamma}\right\} \leqq \mathbf{E}\left\{(1+\varepsilon)\left(\mathbf{E}_{\omega} N_{x}\right)^{\gamma} W_{x}^{\gamma}+c_{\gamma, \varepsilon}\left|\sum_{i=1}^{W_{x}}\left(N_{x}^{(i)}-E_{\omega} N_{x}\right)\right|^{\gamma}\right\} .
$$

As $\mathbf{E}_{\omega} N_{x}=\rho(x)$ is independent of $W_{x}$ we have that

$$
\mathbf{E}\left[\left(\mathbf{E}_{\omega} N_{x}\right)^{\gamma} W_{x}^{\gamma}\right]=\mathbf{E}\left[\rho(x)^{\gamma}\right] E\left(W_{x}^{\gamma}\right)=\left\langle\rho^{\gamma}\right\rangle E\left(W_{x}^{\gamma}\right) .
$$

Under the measure $\mathbf{P}_{\omega}$ the random variables $N_{x}^{(i)}-E_{\omega} N_{x}$ for $i=1,2, \ldots$ are i.i.d. of zero mean and are also independent of $W_{x}$. Therefore, for some universal constant $B_{\gamma}<\infty$, by the Marcinkiewicz-Zygmund inequality

$$
\mathbf{E}_{\omega}\left[\left|\sum_{i=1}^{W_{x}}\left(N_{x}^{(i)}-E_{\omega} N_{x}\right)\right|^{\gamma}\right] \leqq B_{\gamma} \mathbf{E}_{\omega}\left(N_{x}^{\gamma}\right) \mathbf{E}_{\omega}\left(W_{x}^{(\gamma / 2 \vee 1)}\right)
$$

(see [6, Theorem I.5.1]). The independence of the two terms on the right-hand side yields

$$
\mathbf{E}\left[\left|\sum_{i=1}^{W_{x}}\left(N_{x}^{(i)}-E_{\omega} N_{x}\right)\right|^{\gamma}\right] \leqq B_{\gamma} \mathbf{E}\left(N_{x}^{\gamma}\right) \mathbf{E}\left(W_{x}^{(\gamma / 2 \vee 1)}\right) .
$$

Since $\mathbf{E}\left(N_{x}^{\gamma}\right)<\infty$ is independent of $x$, by combining (16), (17) and (18) we have thus shown that for every $\varepsilon>0$ there exists $K_{\gamma, \varepsilon}<\infty$ such that for $x=0,-1, \ldots$,

$$
\mathbf{E}\left(W_{x-1}^{\gamma}\right) \leqq(1+\varepsilon)\left\langle\rho^{\gamma}\right\rangle \mathbf{E}\left(W_{x}^{\gamma}\right)+K_{\gamma, \varepsilon} \mathbf{E}\left(W_{x}^{(\gamma / 2 \vee 1)}\right) .
$$

We deduce next that for every $\gamma \in[1, s)$ there exists $a_{\gamma} \in(0,1)$ and $c_{\gamma}<\infty$ such that for $x=0,-1, \ldots$,

$$
\mathbf{E}\left(W_{x}^{\gamma}\right) \leqq c_{\gamma} a_{\gamma}^{-x}
$$

Indeed, we have already seen that $\mathbf{E}\left(W_{x}\right)=\langle\rho\rangle^{-x}$, hence (20) holds for $\gamma=1$. To see that (20) holds for every integer $\gamma \in[2, s)$ note that then $(\gamma-1) \geqq \gamma / 2 \vee 1$, thus $W_{x}^{(\gamma / 2 \vee 1)} \leqq W_{x}^{\gamma-1}$. Applying a finite induction on $\gamma$ we set $\varepsilon>0$ small enough such that $a_{\gamma}=(1+\varepsilon)\left\langle\rho^{\gamma}\right\rangle \in\left(a_{\gamma-1}, 1\right)$ and define $c_{\gamma}=1+K_{\gamma, \varepsilon} c_{\gamma-1} /\left(a_{\gamma}-a_{\gamma-1}\right)<\infty$. Then, by (19) and the induction hypothesis

$$
\mathbf{E}\left(W_{x-1}^{\gamma}\right) \leqq a_{\gamma} \mathbf{E}\left(W_{x}^{\gamma}\right)+K_{\gamma, \varepsilon} c_{\gamma-1} a_{\gamma-1}^{-x},
$$

which since $W_{0}=1$ results with (20) holding for $\gamma$. Note that $\gamma^{\prime}=\lceil s\rceil-1 \in[1, s)$ is an integer for which (20) holds, and it is left to establish (20) only for $\gamma=\gamma^{\prime}+\eta<s$ such that $\eta \in(0,1)$. Since then $\gamma^{\prime} \geqq \gamma / 2 \vee 1$, we follow the same argument as before with $\gamma^{\prime}$ replacing $\gamma-1$. Fixing $\gamma \in[1, s)$, we have from (20) by monotone convergence that

$$
\mathbf{E}\left(Z^{\gamma}\right)^{1 / \gamma} \leqq \sum_{x=-\infty}^{0} \mathbf{E}\left(W_{x}^{\gamma}\right)^{1 / \gamma}<\infty
$$

as required.

In view of the lower bound of Lemma 2.3, the following upper bound completes the proof of Theorem 1.1. 
Lemma 2.5. For every $v \in\left(0, v_{\alpha}\right)$, if $\gamma<s$ and $n$ is large enough, then

$$
\mathbf{P}\left(X_{n}<v n\right) \leqq n^{1-\gamma}
$$

Proof. Fix $A>-s / \log \langle\rho\rangle$ and let $k=k(n)=A \log n$. On the same probability space on which $\left\{X_{t}\right\}$ is defined, we define the process $\left\{Y_{t}\right\}$ and the hitting times $\tilde{\tau}_{i k}=\min \left\{t \geqq 0: Y_{t}=i k\right\}$, where the only change between $\left\{X_{t}\right\}$ and $\left\{Y_{t}\right\}$ is that for $t \geqq \tilde{\tau}_{i k}, i=0,1, \ldots$, the process $Y_{t}$ is reflected at position $(i-1) k$ (via the use of $\omega_{(i-1) k}=1$ for $\left.t \geqq \tilde{\tau}_{i k}\right)$. Let $N=\lceil v n / k\rceil+1$ and $T_{k}^{(i)}=\tilde{\tau}_{i k}-\tilde{\tau}_{(i-1) k}, i=1, \ldots, N$. Note that $T_{k}^{(i)}$ are identically distributed, each stochastically dominated by $\tau_{k}$. Hence, $\mathbf{E}\left(T_{k}\right) \leqq \mathbf{E}\left(\tau_{k}\right)$. Fix $\lambda \in(1 / s, 1)$. By (11) we know that $\mathbf{E}\left(\tau_{k}^{1 / \lambda}\right)<C k^{1 / \lambda}$ for some $C<\infty$. Moreover, by Hölder's inequality,

$$
\mathbf{E}\left(\tau_{k}\right) \leqq \mathbf{E}\left(T_{k}\right)+\mathbf{P}\left(L_{0} \geqq k\right)^{1-\lambda} \mathbf{E}\left(\tau_{k}^{1 / \lambda}\right)^{\lambda}
$$

Thus by Lemma 2.2, $\mathbf{E}\left(T_{k}\right) / \mathbf{E}\left(\tau_{k}\right) \rightarrow 1$, and therefore Solomon [11, Theorem 1.15] implies that, $\mathbf{E}\left(T_{k}\right) / k \rightarrow v_{\alpha}^{-1}$ for $k \rightarrow \infty$.

On the event that $L_{i k}<k$ for $i=0, \ldots, N$, the two processes $\left\{X_{t}\right\}$ and $\left\{Y_{t}\right\}$ coincide for all $t \leqq \tilde{\tau}_{N k}=\sum_{i=1}^{N} T_{k}^{(i)}$. Moreover, in this case, $\left\{X_{n} \leqq(N-1) k\right\}$ only if $\tilde{\tau}_{N k}>n$. Therefore, by Lemma 2.2 , for all $n$ large enough,

$$
\begin{aligned}
\mathbf{P}\left(X_{n}<v n\right) & \leqq \mathbf{P}\left(X_{n} \leqq(N-1) k\right) \\
& \leqq \mathbf{P}\left(\sum_{i=1}^{N} T_{k}^{(i)}>n\right)+\mathbf{P}\left(\max _{i=0, \ldots, N} L_{i k} \geqq k\right) \\
& \leqq \mathbf{P}\left(\sum_{i=1}^{N} T_{k}^{(i)}>n\right)+n^{-(\gamma-1)} .
\end{aligned}
$$

Since $v^{-1}>v_{\alpha}^{-1}=\lim \sup _{k \rightarrow \infty} k^{-1} \mathbf{E}\left(T_{k}\right)$, there exists $\eta>0$ small enough such that

$$
\begin{aligned}
\mathbf{P}\left(\sum_{i=1}^{N} T_{k}^{(i)}>n\right) & \leqq \mathbf{P}\left(\sum_{i=1}^{N}\left(T_{k}^{(i)}-\mathbf{E}\left(T_{k}\right)\right)>2 \eta n\right) \\
& \leqq 2 \mathbf{P}\left(\sum_{i=1}^{\lceil N / 2\rceil}\left(T_{k}^{(2 i)}-\mathbf{E}\left(T_{k}\right)\right)>\eta n\right) .
\end{aligned}
$$

We now observe that $\left\{T_{k}^{(2 i)}-\mathbf{E}\left(T_{k}\right)\right\}$ is a sequence of i.i.d. mean-zero random variables. Since $T_{k}$ is stochastically dominated by $\tau_{k}$, it follows from (11) that $\mathbf{E}\left(\left|T_{k}-\mathbf{E}\left(T_{k}\right)\right|^{\gamma^{\prime}}\right) \leqq C k^{\gamma^{\prime}}$ for all $\gamma^{\prime} \in(0, s)$. Therefore, by Markov's inequality, for $\gamma^{\prime} \in(\gamma, s)$, and all $n$ large enough,

$$
\mathbf{P}\left(T_{k}-\mathbf{E}\left(T_{k}\right)>\eta n\right) \leqq(\eta n)^{-\gamma^{\prime}} \mathbf{E}\left(\left|T_{k}-\mathbf{E}\left(T_{k}\right)\right|^{\gamma^{\prime}}\right) \leqq n^{-\gamma} .
$$

Hence (see Nagaev [9, (1.3), (1.7a)]),

$$
\mathbf{P}\left(\sum_{i=1}^{\lceil N / 2\rceil}\left(T_{k}^{(2 i)}-\mathbf{E}\left(T_{k}\right)\right)>\eta n\right) \leqq N \mathbf{P}\left(T_{k}-\mathbf{E}\left(T_{k}\right)>\eta n\right)+0.5 n^{1-\gamma} \leqq n^{1-\gamma}
$$




\section{Moments of Total Population Size in BPRE}

Let $M_{1}\left(\mathbb{Z}_{+}\right)$denote the collection of probability measures $\left\{\bar{p}=\{p(i)\}_{i=0}^{\infty}: p(i) \geqq 0\right.$, $\left.\sum_{i} p(i)=1\right\}$ on the nonnegative integers satisfying the further constraint

$$
\rho(\bar{p})=\sum_{i=1}^{\infty} i p(i)<\infty \text {. }
$$

Let the environment $\xi=\left(\xi_{m}\right)_{m \in \mathbb{Z}_{+}}$be an i.i.d. collection of $M_{1}\left(\mathbb{Z}_{+}\right)$-valued random variables with $\alpha$ denoting the law of each $\xi_{m}$. We assume that $\langle\rho\rangle=\int \rho\left(\xi_{0}\right) \alpha\left(d \xi_{0}\right)<1$. For each realization $\xi$ there evolves a population $\left\{W_{m}\right\}_{m \in \mathbb{Z}_{+}}$governed by the laws of the standard temporally non-homogeneous branching process, initialized at $W_{0}=1$. Specifically, let $N_{m}^{(i)}, i=1, \ldots, W_{m}$ be independent random variables, each drawn according to $\xi_{m}$, with $N_{m}^{(i)}$ indicating the number of immediate descendants of the $i^{\text {th }}$ individual at generation $m$. Thus,

$$
W_{m+1}=\sum_{i=1}^{W_{m}} N_{m}^{(i)}
$$

is the size of the population at the $(m+1)^{\text {th }}$ generation. It is well known that the condition $\langle\rho\rangle<1$ implies certain extinction of the BPRE, namely that the total population size

$$
Z=\sum_{m=0}^{\infty} W_{m}
$$

is a.s. finite (see [1, Theorems 1,3]). The RWRE setting we considered in Lemma 2.4 corresponds to a special case of BPRE in which $\xi_{m}(i)=\omega_{-m}\left(1-\omega_{-m}\right)^{i}$ for $i \in \mathbb{Z}_{+}$ is a Geometric distribution, so that $\rho\left(\xi_{m}\right)=\left(1-\omega_{-m}\right) / \omega_{-m}$.

As done in Remark 2.1, since $\left\{N_{m}^{(i)}\right\}_{i}$ are non-negative, identically distributed, and independent of $W_{m}$ we have $\mathbf{E}\left(W_{m+1}\right)=\mathbf{E}\left(W_{m}\right) \mathbf{E}\left(N_{m}\right)$. Then, with $W_{0}=1$ and $\mathbf{E}\left(N_{m}\right)=\langle\rho\rangle<1$, we conclude by monotone convergence that the mean total population size for the BPRE is

$$
\mathbf{E}(Z)=\sum_{m=0}^{\infty} \mathbf{E}\left(W_{m}\right)=\sum_{m=0}^{\infty}\langle\rho\rangle^{m}=(1-\langle\rho\rangle)^{-1}<\infty .
$$

The next lemma supplies moment bounds on the total population size of the BPRE.

Lemma 3.1. Assume that for some $\gamma>1$ both $\left\langle\rho^{\gamma}\right\rangle=\int \rho\left(\xi_{0}\right)^{\gamma} \alpha\left(d \xi_{0}\right)<1$ and

$$
\int\left(\sum_{i=0}^{\infty} i^{\gamma} \xi_{0}(i)\right) \alpha\left(d \xi_{0}\right)<\infty
$$

Then, $C_{\gamma}=\mathbf{E}\left(Z^{\gamma}\right)<\infty$, hence $\mathbf{P}(Z \geqq z) \leqq C_{\gamma} z^{-\gamma}$ for all $z \in \mathbb{N}$.

Proof. Upon setting $m=-x$ and replacing $\omega$ by $\xi$ the proof of Lemma 2.4 applies to the more general setting of the BPRE. By Hölder's inequality, the only requirements of the proof are that $\left\langle\rho^{\gamma}\right\rangle<1$ and that $\mathbf{E}\left(N_{0}^{\gamma}\right)<\infty$. These are exactly the assumptions of Lemma 3.1. 
Remark 3.1. The conditions of Lemma 3.1 are also necessary for $\mathbf{E}\left(Z^{\gamma}\right)<\infty$. Indeed, for $\gamma \geqq 1, \mathbf{E}\left(Z^{\gamma}\right) \geqq \mathbf{E}\left(W_{1}^{\gamma}\right)=\mathbf{E}\left(N_{0}^{\gamma}\right)$, hence $\mathbf{E}\left(Z^{\gamma}\right)=\infty$ when (22) fails. Also, for all $m \in \mathbb{N}$,

$$
\begin{aligned}
\mathbf{E}\left(W_{m+1}^{\gamma}\right) & =\mathbf{E}\left[\mathbf{E}_{\xi}\left(W_{m+1}^{\gamma} \mid W_{m}\right)\right] \geqq \mathbf{E}\left[\left(\mathbf{E}_{\xi} W_{m+1} \mid W_{m}\right)^{\gamma}\right]=\mathbf{E}\left[\left(W_{m} \mathbf{E}_{\xi} N_{m}\right)^{\gamma}\right] \\
& =\mathbf{E}\left[\left(\mathbf{E}_{\xi} N_{m}\right)^{\gamma}\right] \mathbf{E}\left[W_{m}^{\gamma}\right]=\left\langle\rho^{\gamma}\right\rangle \mathbf{E}\left[W_{m}^{\gamma}\right]
\end{aligned}
$$

Hence, $\mathbf{E} W_{m}^{\gamma} \geqq\left\langle\rho^{\gamma}\right\rangle^{m}$ for every $\gamma \geqq 1, m \in \mathbb{N}$. Therefore, $\mathbf{E} Z^{\gamma} \geqq \sum_{m=0}^{\infty} \mathbf{E}\left(W_{m}^{\gamma}\right)$ is infinite for every $\gamma \geqq 1$ such that $\left\langle\rho^{\gamma}\right\rangle \geqq 1$.

\section{Positive and Zero Drifts: Proof of Theorem 1.2}

We shall follow the notations and general outline of the proof of Theorem 1.1, indicating only the changes for the case where $\rho_{\max }=1$ and $\alpha(\{1 / 2\})>0$, which we consider here. For the lower bound on $\mathbf{P}\left(n^{-1} X_{n} \in G\right)$, it again suffices to consider $G=(v-2 \eta, v), 0<2 \eta<v$. Setting $\xi=1-(v-2 \eta) / v_{\alpha}$ we find that

$$
\liminf _{n \rightarrow \infty} n^{-1 / 3} \log \mathbf{P}\left(n^{-1} X_{n} \in(v-2 \eta, v)\right) \geqq \liminf _{n \rightarrow \infty} n^{-1 / 3} \log \mathbf{P}\left(\bar{\tau}_{\eta n}>\xi n\right) .
$$

Let $k=k(n)=\theta n^{1 / 3}$ with $\theta>0$ to be determined below. Using the notations (3) and (4), the conditional probability $\mathbf{P}\left(\bar{\tau}_{k}>\xi_{n} \mid R_{k-1}=0\right)$ is exactly the probability that a simple random walk stays in the interval $[-(k-1),(k-1)]$ for at least $\xi n$ steps. Since $k^{2} n^{-1} \rightarrow 0$, a well-known eigenvalue calculation gives

$$
\lim _{n \rightarrow \infty} k^{2} n^{-1} \log \mathbf{P}\left(\bar{\tau}_{k}>\xi n \mid R_{k-1}=0\right)=-\xi \pi^{2} / 8
$$

(see Spitzer [12, p. 243]). Thus, for all $n$ large enough,

$$
\begin{aligned}
\mathbf{P}\left(\bar{\tau}_{\eta n}>\xi n\right) & \geqq \mathbf{P}\left(R_{k-1}=0\right) \mathbf{P}\left(\bar{\tau}_{k}>\xi n \mid R_{k-1}=0\right) \\
& \geqq \alpha(\{1 / 2\})^{k} e^{-\xi n \pi^{2} /\left(8 k^{2}\right)(1+o(1))} .
\end{aligned}
$$

Recalling that $k=k(n)=\theta n^{1 / 3}$, we see that this lower bound is maximal for

$$
\theta=\left|\frac{\pi^{2} \xi}{4 \log \alpha(\{1 / 2\})}\right|^{1 / 3},
$$

yielding the lower bound

$$
\liminf _{n \rightarrow \infty} n^{-1 / 3} \log \mathbf{P}\left(n^{-1} X_{n} \in(v-2 \eta, v)\right) \geqq-\frac{3}{2} \xi^{1 / 3}\left|\frac{\pi}{2} \log \alpha(\{1 / 2\})\right|^{2 / 3}
$$

(note that (24) remains true with $\xi=1-v / v_{\alpha}$ since $\mathbf{P}\left(n^{-1} X_{n} \in(v-2 \eta, v)\right)$ is nondecreasing in $\eta$ ).

Turning now to the upper bound, we define the process $\left\{Y_{t}\right\}$ as in Lemma 2.5, but now for $k=k(n)=B n^{1 / 3}$, with $B$ a large positive constant. Fixing $v \in\left(0, v_{\alpha}\right)$, 
from (21) and Lemma 2.2 it follows that for every $C_{1}<|\log \langle\rho\rangle|$,

$$
\begin{aligned}
\mathbf{P}\left(X_{n}<v n\right) & \leqq \mathbf{P}\left(\sum_{i=1}^{N} T_{k}^{(i)}>n\right)+e^{-C_{1} B n^{1 / 3}} \\
& \leqq 2 \mathbf{P}\left(\sum_{i=1}^{\lceil N / 2\rceil} T_{k}^{(2 i-1)}>n / 2\right)+e^{-C_{1} B n^{1 / 3}}
\end{aligned}
$$

Recall that $N=\lceil v n / k\rceil+1$ and $\left\{T_{k}^{(2 i-1)}\right\}_{i}$ are i.i.d. with

$$
\mathbf{P}\left(T_{k}^{(1)} \neq \tau_{k}\right) \leqq \mathbf{P}\left(L_{0} \geqq k\right) \leqq e^{-C_{1} B n^{1 / 3}} .
$$

The next lemma supplies tail estimates on $T_{k}=T_{k}^{(1)}$ which are key to the proof of the upper bound.

Lemma 4.1. For every $v^{\prime} \in\left(0, v_{\alpha}\right)$, every $C<\infty, B>0$, and all $n$ large enough,

$$
\mathbf{P}\left(T_{k}>k / v^{\prime}\right) \leqq n^{-C}
$$

where $k=k(n)=B n^{1 / 3}$.

Proof of Lemma 4.1. Fix $v^{\prime} \in\left(0, v_{\alpha}\right)$ and $C<\infty$. By (26) suffices to prove that $\mathbf{P}\left(\tau_{k}>k / v^{\prime}\right) \leqq n^{-C}$. To this end, let $\alpha^{(\eta)}$ be the probability measures on $(0,1)$ obtained from $\alpha$ by moving $\eta>0$ of the mass of $\alpha(\{p\})$ from $p=1 / 2$ to $p=1 / 2-\eta$ (of course $\eta<\alpha(\{1 / 2\})$ ). Fix $\eta>0$ small enough so that both $\int \rho(\omega)^{3 C+1} \alpha^{(\eta)}(d \omega)<1$ and $v^{\prime}<v_{\alpha^{(\eta)}}$. Let $\mathbf{P}^{(\eta)}$ denote the law of the RWRE when the environment $\omega$ is chosen according to the law $\left(\alpha^{(\eta)}\right)^{\mathbb{Z}}$, and $s^{(\eta)}>1$ the corresponding value of $s$. Then, for all $k$,

$$
\mathbf{P}\left(\tau_{k}>k / v^{\prime}\right) \leqq \mathbf{P}^{(\eta)}\left(\tau_{k}>k / v^{\prime}\right) \leqq \mathbf{P}^{(\eta)}\left(X_{\left[k / v^{\prime}\right]}<k\right) \leqq k^{1-s^{(\eta)}+o(1)},
$$

where the last inequality follows from Theorem 1.1 . We conclude by observing that $s^{(\eta)}>3 C+1$ and $k=B n^{1 / 3}$.

Fixing $\varepsilon>0$, let $v(\varepsilon)=\varepsilon v+(1-\varepsilon) v_{\alpha}$ and consider the binomial random variable

$$
W=\sum_{i=1}^{\lceil N / 2\rceil} 1_{\left\{T_{k}^{(2 l-1)}>k / v(\varepsilon)\right\}} .
$$

By Lemma 4.1, for all positive constants $A$ and $C$, the random variable $W$ is stochastically dominated by a $\operatorname{Binomial}\left(n^{3 / 4}, n^{-(A C+1)}\right)$ variable provided that $n$ is large enough. Fix $A$ and let $M=M(n)=n^{1 / 3} /(A \log n)$. It thus follows by Chebycheff's inequality that for $\lambda=C A \log n>0$,

$$
\mathbf{P}(W>M) \leqq e^{-\lambda M}\left(e^{\lambda} n^{-(A C+1)}+1\right)^{n^{3 / 4}} \leqq 2 e^{-C n^{1 / 3}} .
$$

Let $\delta(\varepsilon)=(1-\varepsilon)\left(v_{\alpha}-v\right) /(2 v(\varepsilon))$ and for each subset $I$ of $\{1, \ldots,\lceil N / 2\rceil\}$ of cardinality $M$ let $E_{I}=\left\{\sum_{i \in I} T_{k}^{(2 i-1)}>\delta(\varepsilon) n\right\}$. Since $\left\{\sum_{i=1}^{\lceil N / 2\rceil} T_{k}^{(2 i-1)}>n / 2\right\}$ and 
$\{W \leqq M\}$ imply that at least one of the $E_{I}$ occurs,

$$
\begin{aligned}
\mathbf{P}\left(\sum_{i=1}^{\lceil N / 2\rceil} T_{k}^{(2 i-1)}>n / 2\right) & \leqq \mathbf{P}(W>M)+\sum_{I} \mathbf{P}\left(E_{I}\right) \\
& \leqq 2 e^{-C n^{1 / 3}}+e^{n^{1 / 3} / A} \mathbf{P}\left(\sum_{i=1}^{M} T_{k}^{(2 i-1)}>\delta(\varepsilon) n\right),
\end{aligned}
$$

where the last inequality follows by observing that $E_{I}$ are equally likely and there are at most $n^{M}$ possible subsets $I$.

The random variable $T_{k}$ is stochastically dominated by the random variable obtained when setting $\omega_{x}=1 / 2$ for all $|x|<k$. The latter is the exit time of a simple random walk from the interval $[-(3 k-1),(k-1)]$, which is stochastically dominated by the exit time from the interval $[-(2 k-1),(2 k-1)]$. Hence, for every $\varepsilon>0$, some $c=c(\varepsilon)<\infty$, and all $k$ large enough,

$$
\mathbf{P}\left((2 k)^{-2} T_{k}>x\right) \leqq c e^{-x(1-\varepsilon) \pi^{2} / 8} \quad \forall x \geqq 0
$$

(see Spitzer [12, p. 243]). Consequently, for $\lambda=(1-2 \varepsilon)(2 k)^{-2} \pi^{2} / 8$ and $n$ large enough, by Chebycheff's bound

$$
\begin{aligned}
\mathbf{P}\left(\sum_{i=1}^{M} T_{k}^{(2 i-1)}>\delta(\varepsilon) n\right) & \leqq e^{-\lambda \delta(\varepsilon) n}\left(\mathbf{E}\left(e^{\lambda T_{k}}\right)\right)^{M} \\
& \leqq e^{-(1-3 \varepsilon) \delta(\varepsilon) n^{1 / 3} \pi^{2} /\left(32 B^{2}\right)}
\end{aligned}
$$

Setting $n \rightarrow \infty$ followed by $\varepsilon \downarrow 0$ and $A, C \uparrow \infty$ we see from (25), (27) and (28) that for every $B>0$,

$$
\limsup _{n \rightarrow \infty} n^{1 / 3} \log \mathbf{P}\left(X_{n}<v n\right) \leqq-\min \left\{|\log \langle\rho\rangle| B,\left(1-v / v_{\alpha}\right) \pi^{2} /\left(64 B^{2}\right)\right\}<0 .
$$

\section{Exponentially Unlikely Deviations: Proof of Proposition 1.3}

Step 1. The event $\left\{n^{-1} X_{n} \leqq-\eta\right\}$ implies that the excursion length $L_{0}$ is at least $\eta n$. By Lemma 2.2, for each fixed $\eta>0$, the probability of the latter event decays exponentially in $n$.

Step 2. It suffices to show that

$$
\limsup _{n \rightarrow \infty} n^{-1} \log \mathbf{P}\left(n^{-1} X_{n} \geqq v_{\alpha}+2 \eta\right)<0,
$$

for every $\eta>0$. This is done in two steps. First, we note that a RWRE with positive speed spends finite expected time in the nonpositive half-line; more precisely,

$$
\mathbf{E}\left[\sum_{n=0}^{\infty} \mathbf{1}_{\left\{X_{n} \leqq 0\right\}}\right]=\frac{1+\langle\rho\rangle}{(1-\langle\rho\rangle)^{2}},
$$


provided that $\langle\rho\rangle<1$ (see [11, Theorem 1.19(i)]). Denote the right-hand side of (30) by $C_{\alpha}$. It follows that

$$
\mathbf{E}\left(\tau_{k}-\bar{\tau}_{k}\right) \leqq C_{\alpha}
$$

for any $k \geqq 1$, where $\bar{\tau}_{k}$ was defined in (3).

For the second step, given $\eta>0$, fix $k$ large enough so that

$$
\frac{k}{v_{\alpha}}-C_{\alpha}-2>\frac{k}{v_{\alpha}+\eta} .
$$

By (31) and $E \tau_{k}=k E \tau_{1}=k / v_{\alpha}[11$, Theorem 1.15], this implies that

$$
\mathbf{E}\left(\bar{\tau}_{k}\right)-2>\frac{k}{v_{\alpha}+\eta} .
$$

Consider a modified environment in which $\omega_{i k}$ is set to 1 for all $i$, and at all other integers $x$, the transition probabilities $\omega_{x}$ are still picked according to $\alpha$. Denote by $\bar{\tau}_{k}^{(l)}$ the number of steps the walk in the modified environment, started at $(i-1) k$, takes until it reaches $i k$. Let $\bar{\tau}_{k}^{(i), M}=\bar{\tau}_{k}^{(i)} \wedge M$, where $M$ is large enough such that $\mathbf{E}\left(\bar{\tau}_{k}^{M}\right)>\mathbf{E}\left(\bar{\tau}_{k}\right)-1$. The variables $\bar{\tau}_{k}^{(i), M}$ for $i \geqq 1$ are i.i.d. of bounded support. For each large enough $n$, we can find an integer $m=m(n)$ such that

$$
\frac{n\left(v_{\alpha}+\eta\right)}{k}<m<\frac{n\left(v_{\alpha}+2 \eta\right)}{k}
$$

The inequalities (32) and (33) imply that $n<m\left(\mathbf{E}\left(\bar{\tau}_{k}^{M}\right)-1\right)$. Since the sum $\sum_{i=1}^{m}$ $\bar{\tau}_{k}^{(i), M}$ is stochastically dominated by $\tau_{k m}$, it follows by Cramér's theorem that for some $c>0$,

$$
\begin{aligned}
\mathbf{P}\left(n^{-1} X_{n} \geqq v_{\alpha}+2 \eta\right) & \leqq \mathbf{P}\left(\tau_{k m} \leqq n\right) \\
& \leqq \mathbf{P}\left(\sum_{i=1}^{m} \bar{\tau}_{k}^{(i), M} \leqq m\left(\mathbf{E}\left(\bar{\tau}_{k}^{M}\right)-1\right)\right) \leqq e^{-c m}
\end{aligned}
$$

The assertion (29) follows since the ratio $n / m$ is bounded above as $n \rightarrow \infty$.

Step 3. Assume $\rho_{\max }<1$, namely that $\omega_{\min }=1 /\left(1+\rho_{\max }\right)>1 / 2$. Clearly, it suffices to show that for every $\eta>0$,

$$
\limsup _{n \rightarrow \infty} n^{-1} \log \mathbf{P}\left(n^{-1} X_{n}<(1-4 \eta) v_{\alpha}\right)<0 .
$$

If the trajectory of a nearest neighbor walk on $\mathbb{Z}$ traverses between $x-1$ and $x$ exactly once, we call the latter arrived position a regeneration point and the time following the step between these positions its regeneration time.

Let $Y=\left(Y_{n}\right)_{n \geqq 0}$ denote a simple, asymmetric random walk, which is independent of $\omega$, with $\bar{Y}_{0}=0$, and $\mathbf{P}\left(Y_{n+1}-Y_{n}=1\right)=1-\mathbf{P}\left(Y_{n+1}-Y_{n}=-1\right)=\omega_{\min }$. For every fixed $\omega=\left(\omega_{x}\right)_{x \in \mathbb{Z}}$ construct Bernouli $\left(\left(\omega_{x}-\omega_{\min }\right) /\left(1-\omega_{\min }\right)\right)$ random variables $\xi_{j}(x)$ which are independent of each other and of $Y$. Let $X=\left(X_{n}\right)_{n \geqq 0}$ with

$$
X_{n+1}=X_{n}+\xi_{n}\left(X_{n}\right)+\left(1-\xi_{n}\left(X_{n}\right)\right)\left(Y_{n+1}-Y_{n}\right), \quad X_{0}=0 .
$$


It is easy to see that the process $(X, \omega)$ is identical in law to the RWRE. Furthermore, every regeneration time of $Y$ is also a regeneration time of $X$.

Let $\theta_{0}=0$ and $0 \leqq \theta_{1}<\theta_{2}<\cdots$ denote the regeneration times of $Y$. Denote the corresponding distances of regeneration points by $W_{i}=Y_{\theta_{i+1}}-Y_{\theta_{l}}$ and $Z_{i}=$ $X_{\theta_{l+1}}-X_{\theta_{i}}$. Lemma 5.1 below imply that $\bar{w}=\mathbf{E} W_{1}<\infty$, thus there are a.s. infinitely many regeneration points of $Y$. A direct computation of the joint probability law of $\left(\theta_{i}, Y_{\theta_{l}}\right)_{i=1}^{m}$ reveals that $\left\{W_{i}\right\}_{i=1}^{\infty}$ is a sequence of i.i.d. random variables. A similar computation reveals that the incremental trajectories $\left(Y_{t+\theta_{i}}-Y_{\theta_{i}}: t=\right.$ $0, \ldots, \theta_{i+1}-\theta_{i}$ ) for $i=0,1, \ldots$ are independent and of the same law for each $i \geqq 1$. The random variable $Z_{i}$ is determined by the $i^{\text {th }}$ such trajectory, the environment $\left(\omega_{x}\right)$ for $x \in\left[X_{\theta_{l}}, X_{\theta_{i+1}}\right)$ and the otherwise independent $\left\{\xi_{j}(x)\right\}$. It follows that $\left\{Z_{i}\right\}_{i=0}^{\infty}$ are independent, and identically distributed for $i \geqq 1$. With $\left(X_{\theta_{n}}-X_{\theta_{1}}\right) /\left(\theta_{n}-\theta_{1}\right) \rightarrow v_{\alpha}$ a.s. ([11, Theorem 1.16]), and $\left(Y_{\theta_{n}}-Y_{\theta_{1}}\right) /\left(\theta_{n}-\theta_{1}\right) \rightarrow\left(2 \omega_{\min }-1\right)$ one has that $\bar{z}=\mathbf{E} Z_{1}=\bar{w} v_{\alpha} /\left(2 \omega_{\min }-1\right)<\infty$.

Fix $\eta \in(0,1 / 3)$. Let $k=n(1-3 \eta)\left(2 \omega_{\min }-1\right) / \bar{w}$ and $\delta=\eta /(1-3 \eta)>0$. Then,

$$
\begin{aligned}
\mathbf{P}\left(\theta_{k+1}>n\right) & \leqq \mathbf{P}\left(Y_{\theta_{k+1}}>Y_{n}\right) \\
& \leqq \mathbf{P}\left(Y_{n} \leqq \bar{w} k(1+2 \delta)\right)+\mathbf{P}\left(W_{0}>\bar{w} k \delta\right)+\mathbf{P}\left(\sum_{i=1}^{k} W_{i}>\bar{w} k(1+\delta)\right) .
\end{aligned}
$$

Noting that $\bar{w} k(1+2 \delta)=\left(2 \omega_{\min }-1\right) n(1-\eta)$ it is clear that the first term on the right-hand side of (35) is bounded above by $\exp (-c n)$ for some $c>0$ and all $n$ large. Lemma 5.1 below implies the same type of bound for $\mathbf{P}\left(W_{0}>\bar{w} k \delta\right)$. Lemma 5.1 also guarantees that $\mathbf{E}\left[\exp \left(\lambda W_{1}\right)\right]=\exp (\lambda \bar{w}+o(\lambda))$ for $\lambda>0$ small enough. By Chebycheff's inequality, this leads to

$$
\mathbf{P}\left(\theta_{k+1}>n\right) \leqq e^{-c n}+\mathbf{P}\left(k^{-1} \sum_{i=1}^{k} W_{i}>\bar{w}(1+\delta)\right) \leqq e^{-c_{1} n},
$$

for some $c_{1}>0$ and all $n$ large enough. With $\left\{Z_{i}\right\}_{i \geqq 1}$ positive i.i.d. random variables, of finite mean, and since $k(1-\delta) \bar{z}=n(1-4 \eta) v_{\alpha}$, by Chebycheff's inequality we have for some $c_{2}>0$,

$$
\mathbf{P}\left(n^{-1} X_{n}<(1-4 \eta) v_{\alpha}\right) \leqq \mathbf{P}\left(\theta_{k+1}>n\right)+\mathbf{P}\left(k^{-1} \sum_{i=1}^{k} Z_{l}<(1-\delta) \bar{z}\right) \leqq e^{-c_{2} n} .
$$

The proof of (34) is thus completed by establishing that indeed the distances between regeneration times of the simple, asymmetric random walk $Y$ have exponential tails.

Lemma 5.1. For every $y \geqq d \in \mathbb{N}$,

$$
\mathbf{P}\left(W_{0}+W_{1}>2 y\right) \leqq 2 \mathbf{P}\left(W_{0}>y\right) \leqq 2\left(\rho_{\max }+\rho_{\max }^{d}\right)^{[y / d]-1}
$$

Proof. Fix $y \geqq 1$. Let $\sigma_{y}=\inf \left\{n: Y_{n}=y\right\}$. The event $\left\{W_{0}+W_{1}>2 y\right\}$ implies that either $\left\{W_{0}>y\right\}$ or there is no regeneration point of $Y$ in the interval $[y+1,2 y]$. The latter event is determined by $\left(Y_{n}\right)_{n \geqq \sigma_{y}}$ and by the strong Markov property of $\sigma_{y}$ its probability is exactly $\mathbf{P}\left(W_{0}>y\right)$. To prove the second part of (36), it suffices to consider $y=k d$ with $k \in \mathbb{N}$ and $d \in \mathbb{N}$ such that $\rho_{\max }+\rho_{\max }^{d}<1$. Fixing such 
$d$ we shall prove by induction on $k$ that

$$
\mathbf{P}\left(W_{0}>k d\right) \leqq\left(\rho_{\max }+\rho_{\max }^{d}\right)^{k-1} .
$$

The basis for this induction (the case $k=1$ ) is automatic. Proceeding to the inductive step, we denote the position of the first regeneration point of the killed process $\left(Y_{n}\right)_{n \leqq \sigma_{y}}$ by $W_{0}^{y}$. Observe that $W_{0}^{y} \leqq W_{0}$ and $W_{0}^{y} \in\{1, \ldots, y\}$. Conditioned upon $\left\{W_{0}^{y}=w\right\}$, the event $\left\{W_{0}>y\right\}$ implies that the process $\left(Y_{n}\right)_{n \geqq \sigma_{y}}$ must visit the point $w-1$. Therefore, by the strong Markov property of $\sigma_{y}$,

$$
\mathbf{P}\left(W_{0}>y \mid W_{0}^{y}=w\right) \leqq \mathbf{P}\left(\sigma_{w-1}<\infty \mid Y_{0}=y\right)=\rho_{\max }^{y-w+1}
$$

(use (5) with $\rho(x)=\rho_{\max }$ for all $x$ ). Consequently, for all $k \in \mathbb{N}$,

$$
\begin{aligned}
\mathbf{P}\left(W_{0}>k d\right) & \leqq \sum_{m=1}^{k} \mathbf{P}\left((m-1) d<W_{0}^{k d} \leqq m d\right) \rho_{\max }^{k d-m d+1} \\
& \leqq \sum_{m=1}^{k} \mathbf{P}\left(W_{0}>(m-1) d\right) \rho_{\max }^{(k-m) d+1}
\end{aligned}
$$

which by the induction hypothesis yields,

$$
\mathbf{P}\left(W_{0}>k d\right) \leqq \sum_{m=1}^{k}\left(\rho_{\max }+\rho_{\max }^{d}\right)^{m-2} \rho_{\max }^{(k-m) d+1} \leqq\left(\rho_{\max }+\rho_{\max }^{d}\right)^{k-1} .
$$

This completes the inductive step, and the proof of the lemma.

\section{Concluding Remarks}

1. A setting which we have not covered is that of $\rho_{\max }=1$ while $\alpha(\{1 / 2\})=0$. We believe that in this case, the large deviation probabilities can decay like $\exp \left(-C n^{\beta}\right)$ for any $\beta \in(1 / 3,1)$, with the value of $\beta$ determined by the behavior of the measure $\alpha(\cdot)$ in the neighborhood of $1 / 2$.

2. We conjecture that in the setting of Theorem 1.2,

$$
\lim _{n \rightarrow \infty} n^{-1 / 3} \log \mathbf{P}\left(n^{-1} X_{n} \in G\right)=-\frac{3}{2} \inf _{v \in G}\left(1-v / v_{\alpha}\right)^{1 / 3}\left|\frac{\pi}{2} \log \alpha(\{1 / 2\})\right|^{2 / 3} .
$$

Such a lower bound is given in (24). What is missing is the corresponding (tight) upper bound.

3. Let $0<v<v_{\alpha}$, and suppose that the large-deviation event $n^{-1} X_{n} \in(0, v)$ occurs. We speculate that in this case, the environment $\omega$ is likely to have the following structure:

- When $\rho_{\max }>1$ : one interval of size $(1+o(1))(\log n) / y$ in which the empirical measure of $\omega$ is near $\rho^{s} \alpha$, with the remaining environment as well 
as the actions taken by the random walk being the typical ones (recall that $\left.y=\left\langle\rho^{s} \log \rho\right\rangle\right)$.

- When $\rho_{\max }=1$ and $\alpha(\{1 / 2\})>0$ : one interval of size $(1+o(1)) \theta n^{1 / 3}$ within which $\omega_{x}=1 / 2$ for all $x$, with the remaining environment being a typical one (see (23) for the value of $\theta$ ). We also expect then that the chain behaves typically outside this interval, whereas inside the interval it behaves like a reflected at 0 simple random walk conditioned to stay there for $\left(1-v / v_{\alpha}\right) n$ steps.

In both cases the position of the atypical interval is expected to be roughly uniformly distributed over $(0, v n)$.

To establish such results one has to refine the upper bounds of Sect. 2 and 4 so that if the setting described above is excluded, the upper bound shall be of lower order than the estimates of Theorems 1.1 and 1.2.

4. In the quenched setting a typical environment $\omega$ is fixed. When $\rho_{\max }<1$ or $\rho_{\min }>1$ ("case B" in the terminology of Greven and den-Hollander [5]) the local drifts always point in the same direction, and it is shown in [5] that for almost all environments $\omega$,

$$
I(v)=\lim _{n \rightarrow \infty} \frac{1}{n}\left|\log \mathbf{P}_{\omega}\left(X_{n}=\lfloor v n\rfloor\right)\right|>0 \quad \text { for all } v \neq v_{\alpha} .
$$

On the other hand, when $\rho_{\min }<1 \leqq \rho_{\max }$, ("case $\mathrm{A}$ " in [5]) the random environment has local drifts in both directions and $I(v)=0$ on the whole interval between 0 and $v_{\alpha}$. This subexponential rate is due to the a.s. existence of arbitrarily long stretches in the random environment in which neutral drifts or local potential wells temporarily "trap" the walk, allowing it to slow down. More precisely, in the setting of Theorem 1.1 we shall find for almost every $\omega$ that there is an interval of length $k=(1+o(1)) \log n /(s y)$ in which $k R_{k} \geqq(1+o(1)) \log n / s$ (for example see [3]). The corresponding lower bound on $\mathbf{P}_{\omega}\left(n^{-1} X_{n} \in G\right)$ shall thus be $\exp \left(-n^{1-1 / s+o(1)}\right)$. Similarly, in the setting of Theorem 1.2, by the Erdös-Renýi strong law (for longest run of heads) we shall find for almost every $\omega$ that there is an interval of length $k=\log n /(-\log \alpha(\{1 / 2\}))(1+o(1))$ within which $\omega_{x}=1 / 2$ for every $x$. The corresponding lower bound on $\mathbf{P}_{\omega}\left(n^{-1} X_{n} \in(v-2 \eta, v)\right)$ shall thus be $\exp \left(-K n /(\log n)^{2}(1+o(1))\right)$ with $K=0.5|0.5 \pi \log \alpha(\{1 / 2\})|^{2}\left(1-v / v_{\alpha}\right)$. Since such lower bounds, due to one non-typical segment in the environment, proved to be quite tight in the annealed setting, we expect that the same should apply in the quenched setting.

5. Our assumption that the closed support of $\alpha$ is contained in the open interval $(0,1)$ is used only in the context of Theorem 1.1, where it can be replaced by the weaker assumption of $\left\langle\rho^{s}\right\rangle=1$ for some $s>1$. Indeed, the latter condition suffices for (22) to hold (since $E_{\omega}\left(N_{0}^{\gamma}\right) \leqq k_{\gamma}\left(1+\rho(0)^{\gamma}\right)$ with $k_{\gamma}<\infty$ ), thus for the upper bound to apply. Similarly, this condition suffices for our application of Cramér's theorem enroute to the lower bound of Theorem 1.1.

Acknowledgement. We are indebted to Robin Pemantle for several helpful conversations, especially concerning the proof of the upper bound in Theorem 1.2. 


\section{References}

1. Athreya, K.B., Karlin, S.: "On branching processes with random environments: I. Extinction probabilities”. Ann. Math. Stat. 42, 1499-1520 (1971)

2. Chung, K.L.: Markov Chains with Stationary Transition Probabilities. Berlin: Springer, 1960

3. Dembo, A., Karlin, S.: "Strong limit theorems of empirical functionals for large exceedances of partial sums of i.i.d. variables". Ann. Probab. 19, 1737-1755 (1991)

4. Dembo, A., Zeitouni, O.: Large Deviations Techniques and Applications. Boston: Jones and Bartlett, 1993

5. Greven, A., den Hollander, F.: "Large deviations for a random walk in random environment". Ann. Probab. 22, 1381-1428 (1994)

6. Gut, A.: Stopped random walks. Berlin: Springer, 1988

7. Kesten, H.: "The limit distribution of Sinai's random walk in random environment". Phys. A 138, 299-309 (1986)

8. Kesten, H., Kozlov, M.V., Spitzer, F.: "A limit law for random walk in a random environment". Comp. Math. 30, 145-168 (1975)

9. Nagaev, S.V.: "Large deviations of sums of independent random variables". Ann. Probab. 7, $745-789$ (1979)

10. Sinai, Ya.G.: "The limiting behavior of a one dimensional random walk in a random medium". Theory Probab. Appl. 27, 256-268 (1982)

11. Solomon, F.: "Random walks in random environment". Ann. Probab. 3, 1-31 (1975)

12. Spitzer, F.: Principles of random walk. Berlin: Springer, 1976 
